\title{
Caffeic Acid Effect on Elements of Production Process of Potato Plants (Solanum tuberosum L.) Under Hypothermia
}

\author{
Tamara I. Puzina \\ Department of Botany, Physiology and Biochemistry of Plants \\ Orel State University \\ Orel, Russian Federation
}

\author{
Inna Yu. Makeyeva \\ Department of Botany, Physiology and Biochemistry of Plants \\ Orel State University \\ Orel, Russian Federation
}

\begin{abstract}
The paper investigated the effect of exogenous caffeic acid (0.1 $\mathrm{mM})$ on antioxidant enzyme activity, LPO reactions, the permeability of membranes for electrolytes, the photosynthetic activity and productivity of potato plants (Solanum tuberosum L.) under optimal conditions, as well as under the action of a 2-hour hypothermia of $-2{ }^{\circ} \mathrm{C}$ simulating chilling. The investigation showed stimulation of the activity of SOD, catalase and peroxidase under the influence of caffeic acid. The research on LPO reactions revealed the absence of the caffeic acid effect on the content of hydroperoxides and a decrease in the accumulation of malonic dialdehyde. Caffeic acid stimulated the photochemical activity of isolated chloroplasts (Hill reaction), and induced an increase in plant productivity. Based on the previous data, the authors made an assumption about the role of phytohormones of auxins in changing physiological and biochemical processes caused by the caffeic acid effect. Hypothermia initiated the development of oxidative stress, increased the electrolyte output, and reduced the activity of light reactions of photosynthesis and the plant productivity. Under hypothermia, caffeic acid made a protective influence on the preservation of membranes as a result of stabilizing oxidative stress and, as a consequence, the light reactions of photosynthesis.
\end{abstract}

Keywords-productivity of agricultural plants; exogenous caffeic acid; hypothermia.

\section{INTRODUCTION}

The final productivity of agricultural plants is largely determined by the intensity and the direction of the production process elements, which are primarily represented by photosynthetic activity, respiration, resistance and growth reactions. Currently, researchers offer a variety of ways to regulate them, including both elements of mineral nutrition, biopharmaceuticals and growth regulators.

Modern tendencies in the development of agricultural technologies suggest the use of regulatory substances, which are produced based on plant components. In this connection, the physiological and biochemical role of secondary metabolites, which do not participate in basic metabolism, has become increasingly important $[1,2]$. Phenolic compounds represent the most numerous class of secondary metabolites, which implies the existence of a variety of functions. For a long time, it was believed that they have only inhibitory properties, but over the past decades, the views in this field have undergone drastic changes [3,4]. Research shows that phenolic compounds may have a stimulating effect on growth and organ formation; they are known to participate in the formation of stability, in the work of electron transport chains, in transferring signals in pathogenesis, in forming structural components of the cell wall, as well as in accumulating reserve substances $[5,6]$. The main phenolic compounds in higher plants are phenylpropanoids and flavonoids. It should be noted that the functions of some representatives have been studied to varying degrees. The greatest attention is paid to the study of antioxidant properties of flavonoids $[6,7,8]$. The functions of another large group - phenylpropanoids, which contain hydroxycinnamic acids, in particular, caffeic acid, have been studied to a quite small extent [9]. This applies not only to the optimal environmental conditions but also to the effects of adverse factors.

This study was conducted on potatoes - one of the economically valuable crops. For potato plants, spring chilling $\left(-1{ }^{\circ} \mathrm{C}--2{ }^{\circ} \mathrm{C}\right)$ is fatal at the first stages of vegetation. At the same time, there is no information on the effect of hydroxycinnamic acids on the physiological and biochemical processes of a potato plant under hypothermal conditions.

The aim of the study was to investigate the caffeic acid effect on the antioxidant system, photosynthetic light reactions, the integrity of membranes and the potato plant growth under optimal conditions and in case of a two-hour hypothermia $\left(-2^{\circ} \mathrm{C}\right)$ simulating chilling.

\section{METHODS}

The authors examined potato plants (Solanum tuberosum $L$.) of the Zhukovskii rannii variety, which was selected by the All-Russian Research Institute of Potato farming (Korenevo, Russia). To produce 21-day shoots, the potato tubers, which had been emerged from the state of deep rest, were transferred from the vegetable store, where they had been stored at $+4{ }^{\circ} \mathrm{C}$, into the laboratory conditions $\left(20 \pm 2{ }^{\circ} \mathrm{C}\right)$; then they were sprouted in containers with wet sawdust at first in the dark, and after emergence of shoots on the surface of the substrate, they were grown under room lighting. 
The experiment variants included treatment of plants with $0.1 \mathrm{mM}$ caffeic acid solution (Sigma, USA) by spraying in 15 days after emergence. Control plants were sprayed with water.

Hypothermia was created by placing containers with 21day shoots in a low-temperature case T25/01 (Russia) for 2 hours at $-2{ }^{\circ} \mathrm{C}$. The control plants remained under optimal conditions $20 \pm 2{ }^{\circ} \mathrm{C}$.

Average samples of 21-day shoots were analyzed.

\section{A. The analysis of the work of the antioxidant system}

The catalase activity was determined by the gasometric method according to the amount of oxygen emitted, which was recalculated to the amount of hydrogen peroxide decomposed by catalase.

The peroxidase activity was determined by the time of blue color formation as a result of the benzidine oxidation. To do this, the leaf samples were grounded in acetate buffer $(\mathrm{pH}$ 5.4), decocted, and then centrifuged at $4000 \times \mathrm{g}$ in a laboratory medical centrifuge OPn-8 (Dastane, Kyrgyzstan). A buffer solution and benzidine were added to the centrifuge. Water was added to the control sample, and $0.3 \%$ of hydrogen peroxide solution was added to the experimental sample. The oxidation time of benzidine was measured on the photometer KFK-3-01 (ZOMZ, Russia) at $590 \mathrm{~nm}$.

The superoxide dismutase activity was determined by the reduction reaction of nitrosine tetrazolium, triggered by riboflavin. To do this, the leaf samples were grounded in phosphate buffer $(\mathrm{pH} 7.8)$ and centrifuged at $7000 \times \mathrm{g}$ for 15 minutes. The $2.5 \mathrm{ml}$ reaction medium contained $0.5 \mathrm{ml}$ of superoxide dismutase extract, $50 \mathrm{mM}$ of phosphate buffer, 13 $\mathrm{mM}$ of L-methionine, $75 \mu \mathrm{M}$ of nitroblue tetrazolium, $0.1 \mathrm{mM}$ of EDTA and $2 \mu \mathrm{M}$ of riboflavin. Weighing bottles with reaction medium were placed under a $150 \mathrm{~W}$ lamp. After 15 minutes, the reaction was stopped by turning off the lamp and the optical density of the solutions was measured on the KFK3-01 (ZOMZ, Russia) at $560 \mathrm{~nm}$. The control was an unirradiated reaction mixture.

\section{B. Monitoring of lipid peroxidation reactions (LPO)}

The content of hydroperoxides of lipid fatty acids was evaluated by the reaction of their interaction with ammonium thiocyanate. The leaf samples were grounded in a $0.1 \mathrm{M}$ Tris$\mathrm{HCl}$ buffer solution containing $0.35 \mathrm{M}$ of $\mathrm{NaCl}(\mathrm{pH} 7.6)$, then centrifuged for 1 minute at $2000 \times \mathrm{g}$. $0.4 \mathrm{ml}$ of a $50 \%$ solution of trichloroacetic acid was added to the precipitated protein, filtered and the volume of the extract was adjusted to $10 \mathrm{ml}$ with ethanol. Then, concentrated $\mathrm{HCl}$ and a $5 \%$ solution of Mohr's salt in $3 \% \mathrm{HCl}$ were added. The sample was shaken vigorously and a $20 \%$ solution of ammonium thiocyanate was added. After $10 \mathrm{~min}$, the optical density of the solution at 480 nm was determined.

The content of malonic dialdehyde (MDA) was determined from the color reaction with thiobarbituric acid at heating [10]. To do this, the leaf samples were grounded in a mixture of $0.1 \mathrm{M}$ Tris- $\mathrm{HCl}(\mathrm{pH} 7.6)$ buffer and $0.35 \mathrm{M}$ of $\mathrm{NaCl}$. A $0.5 \%$ solution of thiobarbituric acid in $20 \%$ trichloroacetic acid was added to the extract from the centrifuge $(6000 \times \mathrm{g}, 30 \mathrm{~min})$. The mixture was heated in a water bath for 30 minutes. After rapid cooling, the samples were centrifuged (15 min, $6000 \mathrm{rpm}$ ) and the optical density of the supernatant was measured at $532 \mathrm{~nm}$.

\section{The extent of membrane damage}

The authors determined the output of electrolytes using the method, which had been suggested by Lukatkin [11]. To do this, $10 \mathrm{ml}$ of distilled water were poured onto the leaf samples and left for 4 hours at a room temperature, after which the electrical conductivity was measured on a conductivity meter "Expert 002" (Ekoniks-Expert, Russia). The total output of electrolytes was determined after destruction of the membranes by boiling for 20 minutes. The membrane damage coefficient was then calculated by the formula: $\mathrm{DC}=\frac{L_{D}-L_{0}}{100-L_{0}} \cdot 100 \%$, where $\mathrm{L}_{\mathrm{D}}$ is the output of electrolytes from the plant leaf tissue under hypothermia (\% of the total output of electrolytes), $\mathrm{L}_{0}$ is the output of electrolytes from plant tissues under optimal conditions ( $\%$ of total output of electrolytes).

\section{Photochemical activity of isolated chloroplasts}

Hill reaction was determined according to the amount of potassium ferricyanide, which had been recovered on light. The chloroplast isolation medium contained $0.35 \mathrm{M} \mathrm{NaCl}$ and $0.05 \mathrm{M}$ Tris- $\mathrm{HCl}$ buffer ( $\mathrm{pH} 8)$. The homogenate was filtered and centrifuged twice at $2000 \times \mathrm{g}$ and $8000 \times \mathrm{g}$. After resuspension of $0.035 \mathrm{M} \mathrm{NaCl}$, a suspension of chloroplasts with $0.002 \mathrm{M} \mathrm{K}_{3}\left[\mathrm{Fe}(\mathrm{CN})_{6}\right]$ was placed in weighing bottles. Some of the weighing bottles were exposed (light intensity 20,000 lux) to a specially mounted installation with cooling $\left(18^{\circ} \mathrm{C}\right)$. Some of the weighing bottles were placed to a dark place. The exposure was $10 \mathrm{~min}$. The reaction was extinguished with $5 \%$ TCA. The optical density was measured on KFK-3-01 at $420 \mathrm{~nm}$.

The plant productivity in the soil culture was taken into account by weighing the tubers in each vessel on a technical scale RN-3C13UM (Russia).

\section{E. Statistical processing of the results}

The figures show the arithmetic mean of 5-10 biological replicas and their standard errors. Analytical repetition is 5fold. Using the Student's test, the authors assessed the reliability of the results and considered the differences above 0.95 to be reliable.

\section{RESULTS AND DISCUSSION}

Environmental factors, which deviate in their action from the optimum, cause oxidative stress in the plant organism, which is accompanied by the formation of active forms of oxygen. The antioxidant enzyme of superoxide dismutase acts at the initial stage of plant protection from active forms of oxygen, neutralizing the superoxide radical with the formation of hydrogen peroxide. The results of the study indicate that 2hour hypothermia $\left(-2{ }^{\circ} \mathrm{C}\right)$ to a small extent, but significantly increased the superoxide dismutase activity (Fig. 1). 


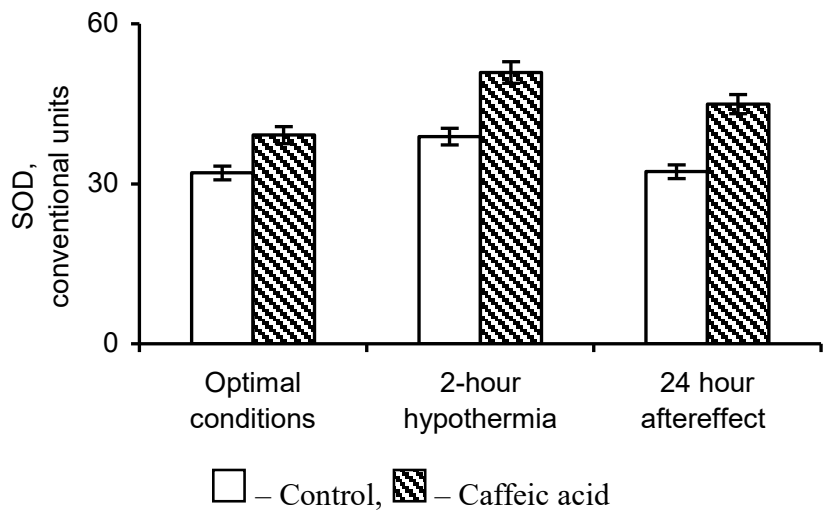

Fig. 1. Superoxide dismutase activity under optimal conditions and hypothermia, depending on the treatment with caffeic acid

Moreover, caffeic acid not only retained a stimulating effect on the superoxide dismutase activity but also increased it in comparison with optimal conditions. A day after the stressor action, the enzyme activity decreased slightly both in the control and in the sample with caffeic acid. However, the stimulating effect of caffeic acid was preserved - an increase in superoxide dismutase activity was 39\%.

As is known, hydrogen peroxide, which is formed mainly in the process of photosynthesis and neutralization of the superoxide radical anion with superoxide dismutase, is utilized by antioxidant enzymes - catalase and peroxidase. Catalase splits hydrogen peroxide into water and molecular oxygen, and peroxidase reduces $\mathrm{H}_{2} \mathrm{O}_{2}$ due to low molecular weight organic compounds. Under hypothermia, both catalase (by $24 \%$ ) and peroxidase (by 59\%) were activated (Fig. 2). In the first 24 hours of aftereffect, catalase activity decreased by $35 \%$, whereas peroxidase activity did not change.

Caffeic acid positively influenced the activity of the studied enzymes under optimal conditions. Perhaps this is due to an increase in the content of endogenous auxins under the caffeic acid influence. The authors had obtained such data earlier [12]. In the literature, there is information that auxins cause the expression of antioxidant enzyme genes and, in particular, catalase [13].

It follows from Fig. 2 that caffeic acid retained its positive effect on the activity of the studied antioxidant enzymes under hypothermia and in the first day of adaptation. The greatest stimulating effect of caffeic acid on the catalase activity was noted in conditions of the stressor's action.
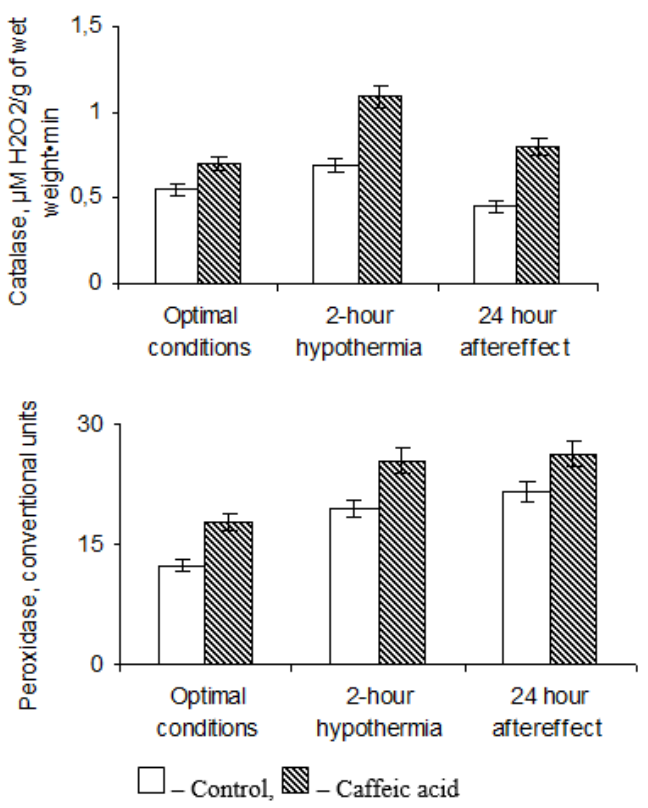

Fig. 2. Influence of caffeic acid on the activity of antioxidant enzymes under the action of hypothermia

Monitoring the reactions of lipid peroxidation on the initial and final products of this accumulation process of lipid hydroperoxides showed that hypothermia had caused an increase in the level of both the hydroperoxides of fatty acids and the malonic dialdehyde. However, a day after the stressor effect, the process slowed down.

The obtained results indicate that, at the optimum temperature, caffeic acid did not affect the initial reactions of LPO, but markedly reduced the accumulation of malonic dialdehyde (by 20\%). Under the conditions of simulated chilling, hypothermia inhibited the accumulation process of hydroperoxides as well as malonic dialdehyde, respectively, by 16 and $24 \%$. Scientists noticed a similar effect of caffeic acid for heat-loving plants, which were grown under conditions of reduced positive temperatures [14]. In the first day of adaptation to the stressor action, this effect of caffeic acid was not observed: the content of hydroperoxides and MDA did not differ from the control sample. The resulting stabilizing effect of caffeic acid on LPO processes appears to be a consequence of the activation of the antioxidant enzymes, examined by the authors (Fig. 3). 

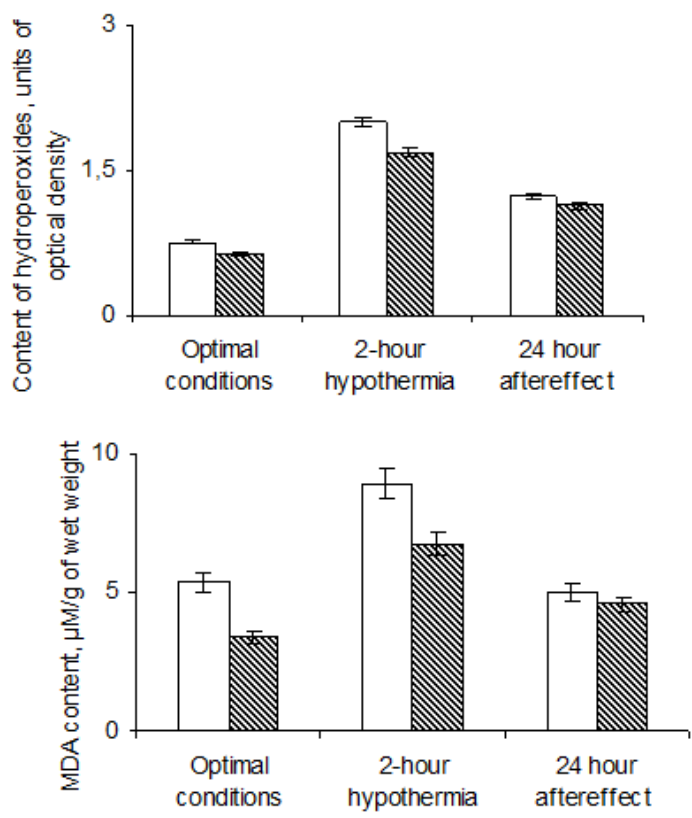

- Control, - Caffeic acid

Fig. 3. Effect of caffeic acid on the LPO process under hypothermia

One of the results of the work of the antioxidant system during the development of oxidative stress is the preservation of the membrane integrity. The membrane permeability under hypothermia was estimated by the electrolyte outflow, determining the electrical conductivity of the solution (Fig. 4).
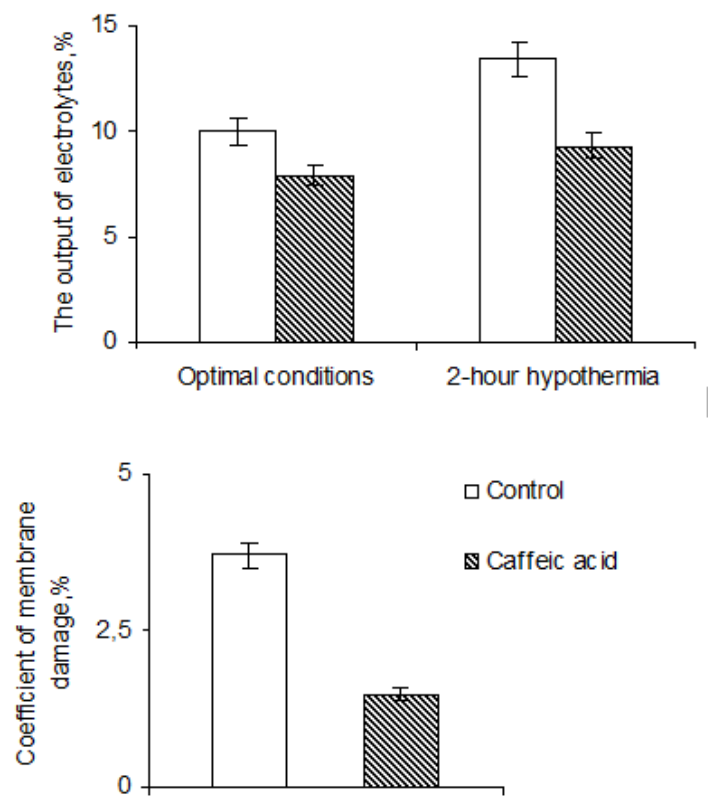

Fig. 4. Effect of caffeic acid on the membrane permeability under hypothermia

Treatment of potato shoots with a $0.1 \mathrm{mM}$ solution of caffeic acid significantly reduced the membrane permeability (by 21\%). A more visible effect (by 30\%) was noted after a 2hour hypothermia action $\left(-2{ }^{\circ} \mathrm{C}\right)$. The calculation of the membrane damage coefficient under hypothermia most clearly demonstrates the protective role of caffeic acid in maintaining the membrane integrity.

The most important component of the production process of plants is photosynthesis. The method of its regulation has both theoretical and practical interest. In the examined literature, the authors could not find information about the effect of hydroxycinnamic acids and, in particular, caffeic acid on the light reactions of the photosynthetic process. The investigation of the photochemical activity of isolated chloroplasts on the reduction of potassium ferricyanide revealed differences, which had been induced by caffeic acid, in the transport speed associated with noncyclic photophosphorylation. Thus, stimulation of this process was $20 \%$ (Fig. 5) and was caused by an increase in the content of endogenous auxins, which are known to increase the content of plastoquinone and the reaction centers of photosystems.

A tendency was observed for a decrease in the chloroplast photochemical activity in potato shoots, which had been exposed to 2-hour hypothermia. However, under these conditions, caffeic acid increased the rate of reduction of ferricyanide to a greater extent (by $35 \%$ ) in comparison with optimal temperature. Apparently, this is due to less damage to chloroplast membranes in this sample against the background of the antioxidant system activation, which is of primary importance for light reactions of photosynthesis.

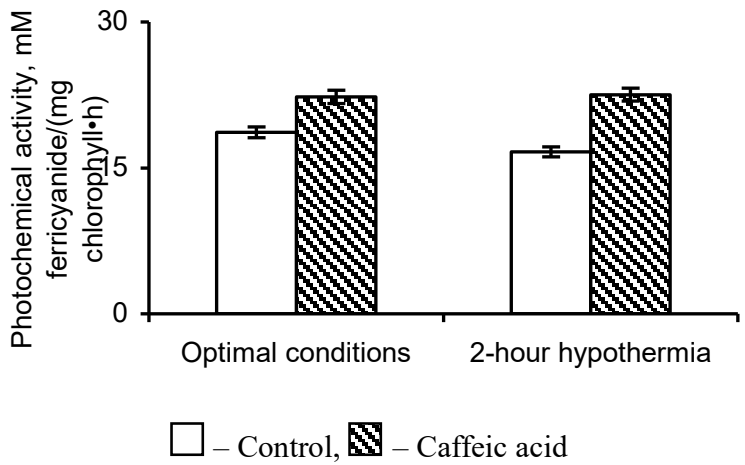

Fig. 5. Effect of caffeic acid on the photochemical activity of isolated chloroplasts under hypothermia

It was of interest to trace the aftereffect of hypothermia on the final productivity of plants. To do this, mother tubers with 15-day shoots, exposed to a 2 -hour hypothermia $\left(-2{ }^{\circ} \mathrm{C}\right)$, were planted in the soil culture in the conditions of the vegetative house on the agrobiological station. A plant was grown in a vessel with $10 \mathrm{~kg}$ of soil and the humidity was maintained at $60 \%$ of the total moisture capacity. The results of Fig. 6 indicate a decrease in plant productivity after the effect of hypothermia, which simulates chilling, by $23 \%$ compared to stress-free conditions. Apparently, this is due to the intensification of LPO reactions, the violation of the membrane integrity and, as a consequence, photosynthetic activity. Caffeic acid, on the contrary, increased potato productivity by $28 \%$ on the background of activation of antioxidant enzymes and reactions of the light phase of photosynthesis. The analysis of the structure of plant productivity showed a decrease in the proportion of large tubers in the sample with hypothermia by $28 \%$. Enrichment of 
plants with caffeic acid increased the mass of large tubers by $30 \%$ in comparison with the control variant. In this case, as the authors have already noted, the positive effect of caffeic acid may be related to an increase in the auxin content. In the previous work [15], it was shown that this group of phytohormones regulates the growth of a potato tuber due to increased outflow of assimilates from the leaves.
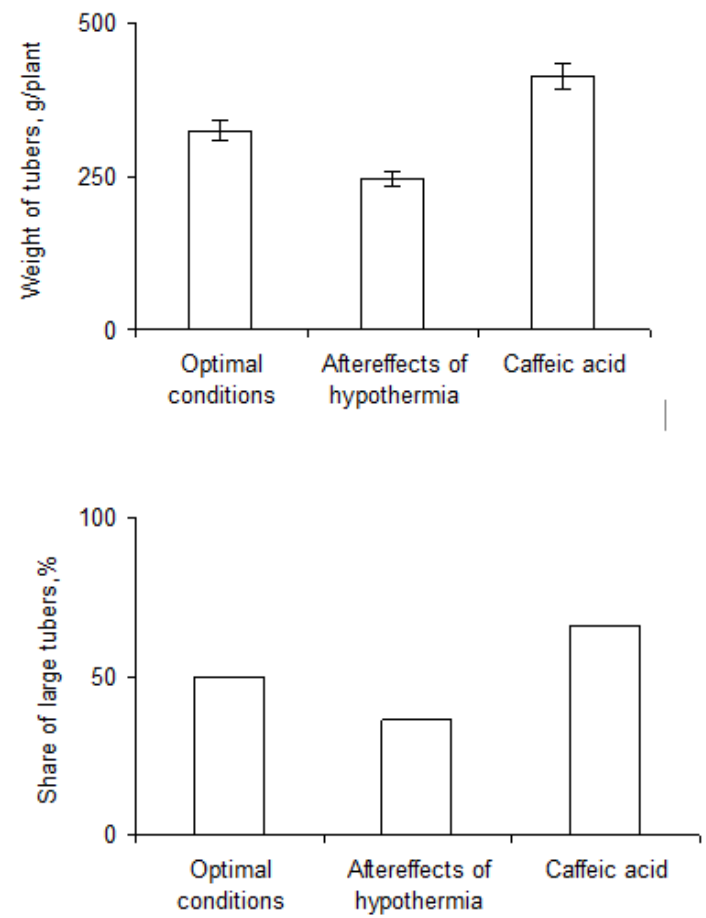

Fig. 6. Effect of hypothermia and caffeic acid on potato plant productivity (soil culture)

\section{CONCLUSION}

The obtained experimental data show that caffeic acid - a representative of hydroxycinnamic acids - participates in the regulation of the studied physiological and biochemical processes, which largely determine the plant productivity. A positive effect was noted in the effect on light reactions of photosynthesis, the antioxidant enzyme activity, as well as the inhibition of LPO reactions. It is assumed that this effect is due to an increase in the content of endogenous auxins. It is noted that a 2 -hour hypothermia $\left(-2^{\circ} \mathrm{C}\right)$, imitating early spring chilling, fatal to potato plants, causes the development of oxidative stress, enhances the membrane permeability for electrolytes, disrupts the electron transport associated with non-cyclic photophosphorylation and, ultimately, reduces the plant productivity. Under hypothermia, caffeic acid retains a positive effect on the photochemical activity of isolated chloroplasts due to a decrease in membrane degradation and antioxidant enzyme activation.

\section{References}

[1] A.M. Nosov, "Functions of plant secondary metabolites in vivo and in vitro," Russian Journal of Plant Physiology, vol. 41, pp. 767-771, 1994.

[2] L. Pan, H.B. Chai, and A.D. Kinghorn, "Discovery of new anticancer agents from higher plants," Frontiers in Bioscience (Scholar Edition), no. 4, pp. 142-156, 2012

[3] V. Lattanzio, P.A. Kroon, S. Quideau, and D. Treutter, "Plant Phenolics - Secondary Metabolites with Diverse Functions," in Recent Advances in Polyphenol Research, vol. 1. Blackwell Publishing Ltd., 2008.

[4] V. Cheynier, G. Comte, K.M. Davies, V. Lattanzio, and S. Martens, "Plant phenolics: Recent advances on their biosynthesis, genetics, and ecophysiology," Plant Physiology and Biochemistry, no. 72, pp. 1-20, 2013.

[5] Y.G. Zuo, C.X. Wang, and J. Zhan, "Separation, characterization, and quantitation of benzoic and phenolic antioxidants in American cranberry fruit by GC-MS," Journal of Agricultural and Food Chemistry, vol. 50, no. 13, pp. 3789-3794, 2002.

[6] A. Fini, C. Brunetti, M. Di Ferdinando, F. Ferrini, and M. Tattini, "Stress-induced flavonoid biosynthesis and the antioxidant machinery of plants," Plant Signaling \& Behavior, vol. 6, no. 5, pp. 709-711, 2011.

[7] O.M. Andersen, and K.R. Markham, Flavonoids. Chemistry, Biochemistry and Applications. New York: CRC Press, 2005, pp. 397441.

[8] E.A. Yagolnik, I.S. Tarakhovskii, I.B. Klenina, S.M. Kuznetsova, E.N Muzafarov, and I.A. Kim, "Study of membranotropic and antioxidant activity of flavonoids and their complexes with ferric iron," Biofizika, no. 58, pp. 819-827, 2013.

[9] R.A. Dixon, and N.L. Paiva, "Stress-induced phenylpropanoid metabolism," Plant Cell, vol. 7, no. 7, pp. 1085-1097, 1995.

[10] A.S. Lukatkin, and V.S. Golovanova, "Lipid peroxidation intensity in chilled leaves of heat-loving plants," Soviet Plant Physiology, no. 35, pp. 610-616, 1988.

[11] A.S. Lukatkin, D.I. Bashmakov, and N.V. Kipaikina, "Protective role of thidiazuron treatment on cucumber seedlings exposed to heavy metals and chilling," Russian Journal of Plant Physiology, no. 50, pp. 305-307, 2003.

[12] T.I. Puzina, and I.Y. Makeeva, "Participation of caffeic acid in regulation of the production process in Solanum tuberosum L.," Agrokhimiya, no. 6, pp. 53-58, 2015.

[13] L.Q.M. Guan, and J.G. Scandalios, "Catalase gene expression in response to auxin-mediated developmental signals," Physiologia Plantarum, no. 114, pp. 288-295, 2002.

[14] Y.Y. Wan, Y. Zhang, L. Zhang, Z.Q. Zhou, X. Li, Q. Shi, X.J. Wang, and J.G. Bai, "Caffeic acid protects cucumber against chilling stress by regulating antioxidant enzyme activity and proline and soluble sugar contents," Acta Physiologiae Plantarum, no. 37, 2015.

[15] T.I. Puzina, I.G. Kirillova, and N.I. Yakushkina, "Dynamics of indole-3acetic acid in the potato organs during various phases of their ontogeny and its role in the regulation of tuber growth," Izvestiya Akademii Nauk Seriya Biologicheskaya, no. 2, pp. 170-177, 2000. 